\title{
ANÁLISIS AGROECONÓMICO DEL USO DE SEMILLA MEJORADA DE MAÍZ EN LOS VALLES ALTOS DE MÉXICO1
}

\author{
Alejandro Espinosa ${ }^{2}$, Miguel A. López $z^{2}$, Margarita Tadeo ${ }^{3}$
}

\begin{abstract}
RESUMEN
Análisis agroeeonómico del uso de semilla mejorada de maíz en los valles altos de México. Se evaluó la capacidad productiva de las generaciones $\mathrm{F}_{1}$ (semilla comercial) y $\mathrm{F}_{2}$ (semilla reciclada) de los híbridos de maíz H-34, H-33, H68, y H-36, generados para los Valles Altos de México por el Centro Nacional de Investigaciones Forestales y Agropecuarias (INIFAP). También se evaluó una cruza simple del CIMMYT y, una variedad de polinización libre del INIFAP (V-23). Se realizó un análisis de rendimientos y de otras características agronómicas de los materiales, y también un análisis económico para determinar la alternativa óptima para los productores referente al uso de semilla durante dos ciclos. En todos los híbridos se presentó una reducción de rendimiento al usar semilla $\mathrm{F}_{2}$ con respecto a la semilla comercial $\mathrm{F}_{1}$, y esta reducción varió desde $5,5 \%$ hasta $51,3 \%$. La mayor reducción de rendimiento por uso de semilla F2 correspondió al híbrido simple $\mathrm{H}-34\left(\mathrm{~F}_{2}\right)$, que sólo produjo $4.327 \mathrm{~kg} / \mathrm{ha}$ comparado con $8.892 \mathrm{kglha}$ para su correspondiente semilla Fl. El rendimiento de la variedad V-23 fue de $7.488 \mathrm{~kg} / \mathrm{ha}$, el cual fue superado estadísticamente por la cruza simple del CIMMYT en su $\mathrm{F}_{1}(9.930 \mathrm{kglha})$ y no tuvo diferencias significativas con respecto a H-34 $\left(\mathrm{F}_{1}\right), \mathrm{H}-33\left(\mathrm{~F}_{1}\right)$ ( $8.887 \mathrm{~kg} / \mathrm{ha}) ; \mathrm{H}-36$ (F) $(9.133 \mathrm{~kg} / \mathrm{ha}) ;$ y la cruza simple del CIMMYT en su $\mathrm{F}_{2}(8.999 \mathrm{~kg} / \mathrm{ha})$. A excepción de ésta última, ningún otro genotipo superó en rendimiento a la variedad. El análisis económico se basó en los resultados del ensayo de rendimientos y en información sobre precios de semilla y de grano, y la tasa de semilla (cantidad de semilla por hectárea). La mejor opción para los productores de maíz de la región, fue el uso de semilla comercial $\left(\mathrm{F}_{2}\right)$ de híbridos dobles en ambos años, ya que proporcionó una tasa de retorno marginal de más del $1.500 \%$.
\end{abstract}

\footnotetext{
1 Trabajo presentado en la XL Reunión Anual del PCCMCA, San José, Costa Rica, 1994.

2 Universidad Nacional Autónoma de México (UNAM), FES-Cuautitlán, México.

3 Centro Internacional de Mejoramiento de Maíz y Trigo (CIMMYT), México.
} 


\section{INTRODUCCIÓN}

En los Valles Altos de México (2.200 - 2.600 msnm), se ha hecho mejoramiento genético en maíz desde 1943, generándose en total 21 híbridos y 13 variedades de polinización libre (VPLs), los primeros en mayor proporción de cruza doble (Cervantes et al., 1987). En la década de los setenta, se orientó el mejoramiento hacia VPLs como una opción para elevar el uso de semilla mejorada, considerando que había cierta renuencia a adquirir semilla, por el precio que este insumo alcanza, por parte de los agricultores. En esa época se obtuvo la mayoría de las VPLs que el Instituto Nacional de Investigaciones Forestales y Agropecuarias (INIFAP) de México ha liberado.

Desde principios de la década de los ochenta, se planteó por los fitomejoradores del INIFAP, el aprovechamiento de híbrido s de cruza simple en los Valles Altos. Por otra parte, el uso de semilla mejorada, aún cuando se ha incrementado notablemente durante los últimos tres años, es muy bajo; siendo frecuente entre los productores de maíz el uso de generaciones avanzadas de semilla híbrida. Esto les permite evitar la necesidad de comprar semilla cada año, la cual tiene precios muy elevados. En la mayoría de estos casos, los agricultores usan semilla de segunda generación (F2), intercambiándo1a o vendiéndola entre los vecinos; pero también se utilizan generaciones más avanzadas (F3, F4 y aún mayor nivel de avance). Esta práctica tiene implicaciones para la producción e ingresos de los productores, ya que se presenta una reducción en la capacidad productiva de los híbridos, debido principalmente al efecto de la pérdida de la heterosis. Esta reducción depende de varios factores, incluyendo el tipo y la constitución genética delluorido y el nivel de endogamia de sus líneas.

La situación actual del maíz en México, es de una alta producción de grano, pero a costos muy elevados debido principalmente a la baja productividad, que no le dan competitividad a nivel internacional. Es indispensable analizar y definir las repercusiones que ocasiona el uso de semilla de generaciones avanzadas en los rendimientos del maíz. Por ello, el objetivo de este trabajo fue evaluar la capacidad productiva de las generaciones F1 (semilla comercial) y F2 (semilla reciclada) de los híbridos generados para Valles Altos por el 00FAP. También se evaluó una cruza simple del CIMMYT y una variedad del INIFAP.

El precio de la semilla mejorada de maíz en los Valles Altos de México es elevado. La semilla de un híbrido de cruza doble alcanza 11,3 veces el precio del grano, y la semilla de VPLs alcanza un precio de 7,6 veces el del grano. Lo anterior señala una cotización elevada de la semilla, lo cual al compararse a nivel internacional es elevado, si se toma el precio de grano como punto de referencia (López-Pereira, 1994; López-Pereira y García, 1994). El uso de semilla mejorada está muy relacionado con la disponibilidad de recursos del agricultor. En un estudio del Programa Nacional de Maíz de Alta Tecnología (PRONAMAT) en México, se determinó que el $92 \%$ de los agricultores maiceros están dispuestos a probar nuevos materiales, pero que la adopción de semilla mejorada se da en mayor proporción entre agricultores que tienen superficies superiores a cinco hectáreas.

El uso de semilla de generaciones avanzadas de híbridos de maíz, es importante en varias regiones de México, especialmente en los Valles Altos, en El Bajío y en el Trópico Húmedo, representando fracciones importantes en relación a la semilla certificada que se emplea (Ortíz, 1990). En algunas zonas, la utilización de este tipo de semilla rebasa el 30\%. Existen evidencias de que el nivel productivo del híbrido desciende cuando se utiliza semilla $\mathrm{F} 2$, ocurriendo la máxima depresión en esta generación, y a niveles de $20-30 \%$ (Espinosa $e t$ al., 1990; Ortíz, 1990). Sin embargo el nivel depende de los genotipos que se manejan y las áreas ecológicas de producción de maíz en el país. En el pasado, se llegó incluso a recomendar el uso de generaciones avanzadas de luoridos en algunas regiones del estado de México, en el estado de Puebla (Molina, 1984), y en la región tropical húmeda (Ramírez et al., 1986). Lo anterior se debía fundamentalmente a la dificultad para tener acceso a semilla certificada. Con los cambios ocurridos en México en materia legal y económica en los últimos años, es conveniente analizar con detalle el factor económico y las ventajas y desventajas del uso de semilla de generaciones avanzadas.

\section{MATERIALES Y MÉTODOS}

\section{Análisis agronómico}

En una localidad representativa de la región de los Valles Altos de México, se evaluaron durante el ciclo Primavera - Verano de 1993, la F1 y F2 de los híbridos H-34, H-33, H-68 Y H-36 (generados por el INIFAP). Para el H-34, también se evaluaron las generaciones F3 y F4 de semilla. Además, se incluyó una cruza simple del CIMMYT y, como testigo, la variedad V-23 del 00FAP. Se empleó un diseño de bloques completos al azar, con cuatro surcos por cinco metros de largo de parcela total y dos surcos de cinco metros de parcela útil. Se analizaron las variables de rendimiento, porcentaje humedad, floración masculina y femenina, y altura de 
planta. Se realizó un análisis de comparación de medias por el método de Tukey al 5\% de probabilidad.

\section{Análisis económico}

El análisis agronómico proporciona importantes resultados sobre las ventajas técnicas de una tecnología o paquete tecnológico a otro, y es muy importante porque ayuda a determinar si existen diferencias significativas entre las alternativas, y a identificar las que serían más aceptables desde el punto de vista agronómico para los agricultores. Para decidir la alternativa tecnológica más aceptable para el agricultor, es necesario complementar el análisis agronómico con un análisis económico marginal de las alternativas. Las bases teóricas del análisis económico marginal de tecnologías agrícolas, se encuentran en CIMMYT (1988); y un ejemplo práctico de su aplicación puede encontrarse en López-Pereira y Espinosa-Calderón (1993). La regla principal del análisis es que, si la tasa de retorno marginal (CIMMYT, 1988), que resulta de cambiarse de una tecnología A a una tecnología B es mayor que la tasa mínima de retorno aceptable por el agricultor, entonces la tecnología B es preferida. En este caso, se analizaron cinco alternativas relacionadas con el uso de semilla comercial y de generaciones avanzadas de maíz durante dos ciclos de cultivo:

$\mathrm{T} 1=\mathrm{V}-23$ (VPLs mejorada).

Semilla comercial el primer ciclo y reciclada el segundo.

$\mathrm{T} 2=\mathrm{H}-33$ (Híbrido doble).

Semilla comercial $\left(\mathrm{F}_{1}\right)$ el primer ciclo y reciclada $\left(\mathrm{F}_{2}\right)$ el segundo.

T3 = H-34 (Híbrido simple).

Semilla $F_{1}$ el primer ciclo y $F_{2}$ el segundo.

$\mathrm{T} 4=\mathrm{H}-33$

Semilla $\mathrm{F}_{1}$ ambos ciclos.

$\mathrm{T} 5=\mathrm{H}-34$.

Semilla $F_{1}$ ambos ciclos.

Para el análisis económico marginal realizado en este caso, se asume que el único in sumo que varía entre las alternativas es la semilla de maíz. Es decir, todas las otras actividades en la producción del cultivo son iguales para todas las alternativas, y se asume que esto también se aplica para la forma en que se realizó el ensayo agronómico, Para realizar el análisis marginal, se necesita información sobre precios de semilla, de grano y la tasa de semilla usada (kg por hectárea). Toda la información económica usada en el análisis corresponde a la región de Valles Altos de México. Otra información importante en el análisis, son los rendimientos obtenidos para cada una de las alternativas en el ensayo agronómico, los cuales son usados para determinar los ingresos por cada alternativa. Los rendimientos obtenidos de ensayos en estaciones experimentales normalmente deben ser ajustados hacia abajo, para tomar en cuenta las posibles reducciones en situaciones de producción de maíz en fincas de agricultores. Finalmente, como se trata de un análisis de dos períodos, es necesario usar una tasa de descuento para hacer los valores comparables en un punto en el tiempo.

\section{RESULTADOS Y DISCUSIÓN}

Para todas las variables evaluadas en el análisis agronómico, se presentaron diferencias altamente significativas para el factor de variación entre tratamientos, no así para repeticiones. El coeficiente de variación para rendimiento fue $10,3 \%$, con una media de $6.148 \mathrm{~kg} / \mathrm{ha}$. En todos los híbrido s se presentó una reducción de rendimiento al usar semilla reciclada o F2, con respecto a la semilla comercial o F1, y esta reducción varió desde 5,5\% hasta 51,3\%. La mayor reducción por uso de semilla F2 correspondió al híbrido simple $\mathrm{H}-34$, que sólo rindió $4.327 \mathrm{~kg} / \mathrm{ha}$ comparado con $8.892 \mathrm{~kg} / \mathrm{ha}$ para su correspondiente semilla F1 (Cuadro 1). El H-34 es el primer híbrido simple desarrollado especialmente para los Valles Altos de México. Este híbrido fue liberado en 1990 y sus líneas son de baja endogamia, S3 y S 1 (Espinosa, et al., 1990). Debido a su bajo rendimiento de semilla, el costo de producción de este híbrido es muy elevado, especialmente con respecto a los híbridos dobles, y el precio de esta semilla es muy por arriba de cualquier otro material. El rendimiento de la variedad V-23 fue $7.488 \mathrm{~kg} / \mathrm{ha}$, el cual fue superado estadísticamente por la cruza simple experimental del CIMMYT en su F1 (9,930 kg/ha) y no presentó diferencias estadísticas significativas con H-34 (F1), H-33 (F1) con 8.887 kg/ha; H-36 (F1) con 9.133 $\mathrm{kg} / \mathrm{ha}$; y con la cruza simple del CIMMYT en su F2 $(8.999 \mathrm{~kg} / \mathrm{ha})$. A excepción de esta última, ningún otro genotipo superó en rendimiento a la variedad al usar semilla de generaciones avanzadas (F2, F3 ó F4). Puede notarse también, que se presenta una fuerte reducción en el rendimiento por el uso de semilla F2, y que el rendimiento se estabiliza o se recupera un poco con generaciones F3 y más avanzadas, pero a niveles muy por debajo de los de la semilla F1 (ver los híbridos H-34 y M17 x M18 en el Cuadro 1). Este efecto puede también notarse en las otras características agronómicas, como son días a floración y altura de planta. El contenido genético del material, es un factor importante en la disminución de rendimiento que se presenta al usar semilla F2. Puede notarse que los híbridos H-68 y la cruza 
Cuadro 1. Comparación de medias (Tukey $=0,05$ de probabilidad) para variables evaluadas en híbridos de maíz de Valles Altos de México, 1993.

\begin{tabular}{|c|c|c|c|c|c|c|}
\hline \multirow{2}{*}{ Material } & \multirow{2}{*}{ Tipo $^{\mathbf{a}}$} & \multirow{2}{*}{$\begin{array}{c}\text { Rendimiento } \\
(\mathrm{kg} / \mathrm{ha})\end{array}$} & \multirow{2}{*}{$\%$ vs la $\mathbf{F}_{\mathbf{l}}^{\mathbf{b}}$} & \multicolumn{2}{|c|}{ Días a floración } & \multirow{2}{*}{$\begin{array}{c}\text { Altura de } \\
\text { planta }(\mathrm{cm})\end{array}$} \\
\hline & & & & Masc. & Fem. & \\
\hline H-34 (F1) & CS (be) & $8.892 \mathrm{ab}$ & 100 & $84 \mathrm{def}$ & $87 \mathrm{cdef}$ & 243 bcde \\
\hline H-34 (F2) & CS (be) & $4.327 \mathrm{fg}$ & 49 & $95 \mathrm{ab}$ & $98 \mathrm{~b}$ & $265 \mathrm{ab}$ \\
\hline H-34 (F3) & CS (be) & $4.748 \mathrm{ef}$ & 53 & $86 \mathrm{~cd}$ & $90 \mathrm{c}$ & 234 bcdefg \\
\hline H-34 (F4) & CS (be) & $5.678 \mathrm{def}$ & 64 & $83 \mathrm{def}$ & $89 \mathrm{~cd}$ & 242 bcdef \\
\hline H-33 (F1) & $\mathrm{CD}$ & $8.887 \mathrm{ab}$ & 100 & 83 defg & 85 cdefg & 249 bcd \\
\hline H-33 (F2) & $\mathrm{CD}$ & 6.513 cde & 73 & 84 def & $87 \mathrm{cdef}$ & $230 \mathrm{bcdefg}$ \\
\hline H-68 (F1) & CS (me) & 6.404 cde & 100 & 77 hi & $80 \mathrm{fgh}$ & 231 bcdefg \\
\hline H-68 (F2) & CS (me) & $6.050 \mathrm{cdef}$ & 94 & $76 \mathrm{hi}$ & $79 \mathrm{gh}$ & 213 defgh \\
\hline H-36 (F1) & $\mathrm{CS}(\mathrm{ae})$ & $9.133 \mathrm{ab}$ & 100 & 84 def & $86 \mathrm{cdef}$ & 237 bcdef \\
\hline H-36 (F2) & CS (ae) & 6.481 cde & 71 & 80 efgh & 83 defgh & 226 bcdefg \\
\hline CIMMYT (F1)c & CS (ae) & $9.930 \mathrm{a}$ & 100 & $88 \mathrm{~cd}$ & 88 cde & 216 cdefgh \\
\hline CIMMYT (F2) & CS (ae) & $8.999 \mathrm{ab}$ & 91 & $90 \mathrm{~cd}$ & $90 \mathrm{c}$ & 208 efgh \\
\hline M17xM18 (FD & CS (be) & $7.859 \mathrm{bc}$ & 100 & 84 def & $86 \mathrm{cdef}$ & 223 cdefg \\
\hline M17xM18 (F2) & CS (be) & $4.916 \mathrm{ef}$ & 63 & $87 \mathrm{de}$ & $90 \mathrm{c}$ & $201 \mathrm{fgh}$ \\
\hline M17xM18 (F3) & CS (be) & $5.018 \mathrm{ef}$ & 64 & 86 cde & $88 \mathrm{~cd}$ & 211 defgh \\
\hline $\mathrm{V}-23$ & VPL & $7.488 \mathrm{bcd}$ & 100 & $87 \mathrm{~cd}$ & $90 \mathrm{c}$ & $302 \mathrm{a}$ \\
\hline DSH (0.05) & & 2.001 & & 6 & 7 & 41 \\
\hline
\end{tabular}

${ }^{\text {a }}$ CS = Híbrido de cruza simple; $\mathrm{CD}$ = híbrido de cruza doble; VPL = variedad de polinización libre; be, me, ae $=$ niveles bajo, medio y alto de endogamia de las líneas que forman los híbridos, respectivamente.

b Porcentaje de cambio rendimiento con semilla de las generaciones avanzadas con respecto a su correspondiente $\mathrm{F}_{1}$.

c Cruza simple experimental del CIMMYT.

simple del CIMMYT sufrieron reducciones muy pequeñas $(<10 \%)$, mientras que en el $\mathrm{H}-34$ fue muy fuerte la reducción. El efecto de rendimiento de la semilla F2 del híbrido de cruza doble, fue una reducción del $27 \%$ con respecto al de la semilla F1, que es dentro del rango reportado para este tipo de híbridos.

En el análisis económico, se analizaron sólo algunos de los genotipos comerciales, para determinar la secuencia de uso de semilla de maíz más aceptable para el productor en un período de dos años. Las alternativas planteadas fueron el uso de semilla de la VPL (V-23), del híbrido doble H-33; y del híbrido simple H-34. Se asumió que se usa semilla comercial de $\mathrm{V}-23$ el primer ciclo, y reciclada el segundo. Para los híbridos, se consideraron las alternativas de usar semilla F1 en ambos ciclos o F1 en el primer ciclo y F2 en el segundo. El resultado del análisis marginal se presenta en el Cuadro 2.

La mejor opción económica para los productores de maíz de los Valles Altos de México, es el uso de semilla F1 del híbrido doble $\mathrm{H}-33$ en ambos años (alternativa T4, ver Cuadro 2). Esta alternativa proporciona la última tasa de retorno marginal (TRM) que es superior a una tasa de retorno mínima aceptable por agricultores $(1,933 \%)$ (CIMMYT, 1988). Por su alto rendimiento, el uso de semilla $\mathrm{F} 1$ en el primer ciclo y F2 en el segundo ciclo del híbrido doble H-33 (T2) es también una alternativa atractiva, especialmente cuando se compara con el uso de la variedad V-23 (TI). Este cambio de la alternativa $\mathrm{T} 1$ a la $\mathrm{T} 2$, resultaría en una TRM $384 \%$, que también parece ser superior al mínimo aceptable. Debe enfatizarse, sin embargo, que la alternativa óptima es el uso de semilla F1 del híbrido doble en ambos ciclos (T4). Los agricultores que sólo cambian de TI a T2, dejan de percibir la mayor parte de los beneficios ofrecidos por la semilla híbrida comercial, y deben realizar el cambio directo de T1 a T4, para maximizar sus ganancias. Por su elevado precio, baja diferencia de rendimiento con respecto al híbrido doble, y marcada reducción de rendimiento cuando se usa semilla F2 el uso de semilla F1 y F2 del híbrido simple H-34 (T3), no es una alternativa económicamente aceptable. Finalmente, el cambio de T4 a T5 proporciona una TRM muy baja (4\%), la cual no sería aceptable para los agricultores de la región, y esto elimina económicamente la alternativa de usar semilla F1 de híbrido s simples en ambos ciclos. 
Cuadro 2. Análisis económico marginal del uso de semilla mejorada de maíz en los Valles Altos de México, 1993.

\begin{tabular}{|c|c|c|c|c|c|}
\hline & \multicolumn{5}{|c|}{ Alternativas de uso de semilla durante dos ciclos ${ }^{a}$} \\
\hline & TI & $\mathbf{T} 2$ & T3 & T4 & T5 \\
\hline Costos de semilla ${ }^{b}$ & \multicolumn{5}{|c|}{$(\mathrm{N} \$ / \mathrm{ha})$} \\
\hline Primer ciclo & 100 & 180 & 260 & 180 & 260 \\
\hline Segundo ciclo & 19 & 19 & 19 & 180 & 260 \\
\hline Total costos que varían & 117 & 197 & 277 & 344 & 496 \\
\hline Costo marginal & & 80 & 80 & 67 & 152 \\
\hline \multicolumn{6}{|l|}{ Ingresos $^{c}$} \\
\hline Primer ciclo & 4.493 & 5.332 & 5.335 & 5.332 & 5.335 \\
\hline Segundo ciclo & 4.493 & 3.908 & 2.596 & 5.332 & 5.335 \\
\hline Total & 8.577 & 8.884 & 7.695 & 10.179 & 10.185 \\
\hline \multirow[t]{2}{*}{ Ingreso marginal } & & 307 & -1.189 & 1.295 & 6 \\
\hline & \multicolumn{5}{|c|}{$(\%)$} \\
\hline Tasa de retorno marginald & & 384 & $\mathrm{D}$ & 1.933 & 4 \\
\hline
\end{tabular}

a Las tecnologías son las siguientes:

$\mathrm{TI}=\mathrm{V}-23$ (la VPL), semilla comercial en el primer ciclo y reciclada en el segundo.

$\mathrm{TI}=\mathrm{H}-33$ (híbrido de cruza doble), semilla comercial $\left(\mathrm{F}_{1}\right)$ en el primer ciclo y reciclada $\left(\mathrm{F}_{2}\right)$ en el segundo.

$\mathrm{T} 3=\mathrm{H}-34$ (híbrido de cruza simple), semilla $\mathrm{F}_{1}$ en el primer ciclo y $\mathrm{F}_{2}$ en el segundo.

$\mathrm{T} 4=\mathrm{H}-33$, semilla $\mathrm{F}_{1}$ en ambos ciclos.

$\mathrm{T} 5=\mathrm{H}-34$, semilla $\mathrm{F}_{1}$ en ambos ciclos.

b Basado en una tasa de semilla de $20 \mathrm{~kg} / \mathrm{ha}$; precio de semilla comercial de la variedad V-23 de N\$5/kg, del híbrido doble H-33 de N\$9/kg, y del híbrido simple H-34 de N\$13/kg.; la semilla reciclada valorada en N\$ $0,94 / \mathrm{kg}$, lo cual es $25 \%$ mayor que el precio del grano. Los valores del segundo ciclo fueron descontados por un año a una tasa del $10 \%$. Se asume que los costos de semilla son los únicos que varían entre las diferentes alternativas. Los valores están dados en nuevos pesos mexicanos (tasa de cambio: N\$3,1/ US\$ 1 en Dic. 1993).

c Basado en los rendimientos del Cuadro 2 (ajustados hacia abajo en 20\%); y precio del grano de N\$ 0,75/kg. Los valores del segundo ciclo fueron descontados por un año a una tasa del $10 \%$.

d La tasa de retorno marginal resulta de dividir los ingresos marginales entre los costos marginales, y expresar el resultado en porcentaje (después de ordenar las alternativas en forma ascendente de Total de Costos que Varían). La alternativa T3 es Dominada (D) porque sus ingresos marginales con respecto a la T2 son negativos, y queda eliminada del análisis marginal.

En resumen, tanto el análisis agronómico como el económico indican que el uso de generaciones avanzadas de semilla híbrida, no conviene a los productores de la región. Esto de hecho se ha traducido en la eliminación del H-34 en los programas de producción de semillas de las empresas semilleras de los Valles Altos de México, y probablemente dificultaría la explotación comercial de otros híbridos simples (H-68 y H-36), así como otros materiales no evaluados en este trabajo. El precio de la semilla juega un papel fundamental en la decisión por los productores de usar semilla comercial o de generaciones avanzadas de un híbrido (Byerlee, Morris, y López-Pereira, 1993), y este precio está influenciado principalmente por los costos de producción de la semilla. Los materiales que pueden ser producidos a bajo costo son importantes para mantener precios de semilla aceptables, con lo cual se puede incrementar el uso de semilla comercial y al mismo tiempo reducir el uso de generaciones avanzadas de semilla híbrida. En este sentido, el híbrido doble H-33 presenta características favorables para producción de grano, y es ya ellu'brido de mayor uso por las empresas semilleras en la región.

\section{LITERATURA CITADA}

BYERLEE, D.; M.L. MORRIS; M.A. LÓPEZ-PEREIRA. 1993. "Hybrid maize and the small-scale farmer: economic and policy issues." Documento presentado en el V Taller Regional de Maíz en Asia. Hanoi y Ho Chi Minh City: República Socialista de Vietnam. 35 p.

CERVANTES R.; RODRÍGUEZ V J., J.; GUEVARA C. J. 1987. "Listado de variedades liberadas por el INIA de 1942-1985." Publicación especial No. 122. México, D.E: INIFAP. 71 p. 
CIMMYT. 1988. La formulación de recomendaciones a partir de datos agronómicos: un manual metodológico de evaluación económica. Edición completamente revisada. México D.F: CIMMYT. 80 p.

ESPINOZA C.; TADEO R. A., M.; VALDIVIA B. R. 1990. "Capacidad productiva de semilla F1, F2 y F3 del híbrido simple de maíz H-34." In Resúmenes del XIII Congreso Nacional de la Sociedad Mexicana de Fitogenética, ESAHE. Cd. Juárez, Chihuahua, México. 371 p.

LÓPEZ-PEREIRA, M.A.; ESPINOSA CALDERÓN, A. 1993. "Análisis económico de la producción y uso de semilla mejorada de maíz: El caso de México." Documento presentado en la XXXIX Reunión Anual del PCCMCA. Guatemala, Guatemala. 15 p.

LÓPEZ-PEREIRA, M. A. 1994. "Las industrias de semilla de maíz de Centro América y México: Relaciones entre los sectores público y privado." Documento presentado en la XL Reunión Anual del PCCMCA. San José, Costa Rica. $21 \mathrm{p}$.
LÓPEZ-PEREIRA, M.A.; J.C. GARCIA. 1994. "Las industrias de semilla de maíz de Brasil y México: Desarrollo histórico, temas de actualidad y prospectos para el futuro." Borrador. Programa de Economía. México D.E CIMMYT. 123 p.

MOLINA M., J.c. 1984. Aprovechamiento de las generaciones avanzadas de maíz (Zea mays L.) H-133 en Valse-quillo, Puebla. Tésis Profesional. Chapingo, México: UACH. 87 p.

ORTÍZ T., E. 1990. Rendimiento de híbridos de maíz (Zea mays L.) de la zona de transición El Bajío-Valles Altos, por efecto de la utilización de semilla de generaciones $\mathrm{F}_{1}$ y $\mathrm{F}_{2}$. Tesis profesional. Chapingo, México: UACH. $62 \mathrm{p}$.

RAMÍREZ V., P., BALDERAS M., M.; GERÓN X, E 1986. "Potencial productivo de las generaciones avanzadas de los híbridos tropicales de maíz H-503, H-507 y H-510." Fitotecnia 8:20-34. 